\title{
Superconducting Atomic Contacts inductively coupled to a microwave resonator
}

\author{
C. Janvier ${ }^{1}$, L. Tosi ${ }^{2}$, Ç. Ö. Girit ${ }^{1}$, M. F. Goffman ${ }^{1}$, H. \\ Pothier $^{1}$, C. Urbina ${ }^{1}$. \\ ${ }^{1}$ Quantronics Group, Service de Physique de l'État Condensé (CNRS, URA 2464), \\ IRAMIS, CEA-Saclay, 91191 Gif-sur-Yvette, France \\ ${ }^{2}$ Centro Atómico Bariloche and Instituto Balseiro, Comisión Nacional de Energía \\ Atómica (CNEA), 8400 Bariloche, Argentina \\ E-mail: cristian.urbina@cea.fr
}

\begin{abstract}
We describe and characterize a microwave setup to probe the Andreev levels of a superconducting atomic contact. The contact is part of a superconducting loop inductively coupled to a superconducting coplanar resonator. By monitoring the resonator reflection coefficient close to its resonance frequency as a function of both flux through the loop and frequency of a second tone we perform spectroscopy of the transition between two Andreev levels of highly transmitting channels of the contact. The results indicate how to perform coherent manipulation of these states.
\end{abstract}

Keywords: break junctions, atomic contacts, superconductivity, Josephson effect, Andreev states. 


\section{Introduction}

Atomic-size contacts between metallic electrodes are routinely obtained using either scanning tunneling microscopes or break-junctions [1]. From the electrical transport point of view, atomic contacts are simple systems. First, as for any good metal, electronelectron interactions are strongly screened. Second, because their transverse dimensions are of the order of the Fermi wavelength (typically $0.2 \mathrm{~nm}$ ) they accommodate only a small number of conduction channels. Moreover, as the transmission probability $\tau_{i}$ for electrons through each conduction channel can be adjusted and measured in-situ [2], atomic contacts provide a test-bed to explore mesoscopic electronic transport [3, 4, 5]. In particular, when the metal is a superconductor atomic-contacts constitute elementary Josephson weak-links that allow probing the foundations of the Josephson effect [6].

Josephson supercurrents [7] will flow through any barrier weakly coupling two superconductors, including a tunnel junction, a constriction, a molecule, or a normal metal [8]. Microscopically, weak links differ in their local quasiparticle excitation spectrum. For a non-interacting system, this spectrum is determined by the length of the weak link, compared to the superconducting coherence length, and its configuration of conduction channels as characterized by the set of transmission probabilities $\left\{\tau_{i}\right\}$. The excitation spectrum of a short single-channel weak link of arbitrary transmission $\tau$ contains, besides the continuum of states at energies larger than the superconducting gap $\Delta$, a sub-gap spin-degenerate level, known as the Andreev doublet (Figure 1). Its energy $E_{A}=\Delta \sqrt{1-\tau \sin ^{2}(\delta / 2)}[9,10]$ is a $2 \pi$-periodic function of the superconducting phase difference $\delta$ across the weak link. It is precisely this phase dependence that gives rise to the Josephson supercurrent. In the widespread case of Josephson tunnel junctions, for which all $\tau_{i} \ll 1, E_{A i} \sim \Delta$ and the lowest-lying excitations conserving electron parity have a threshold energy only slightly lower than $2 \Delta$. By absorbing energy $\gtrsim 2 \Delta$ a pair can be broken and two quasiparticles created at essentially the gap energy $\Delta$, like in a bulk superconductor.

Two different spectroscopy experiments have recently probed this excitation spectrum for superconducting atomic contacts containing channels of high transmisssion. The first experiment [11, 12] spotlighted the lowest energy excitation that conserves electron parity, the "Andreev transition" of energy $2 E_{A}$, which leaves two quasiparticles in the Andreev level (red double arrow in Figure 19). The second experiment [13] revealed a second type of excitation, with minimum energy $E_{A}+\Delta$. In this case, a localized Andreev pair is broken into one quasiparticle in the Andreev level and one in the continuum (green arrows in Figure 19) [14, 15, 16], thus leaving the system in an "odd" state. These odd states had been previously detected through the spontaneous trapping of a single out-of-equilibrium quasiparticle in the Andreev doublet [17]. This ensemble of results firmly support the description of the Josephson effect in terms of Andreev bound states.

If parity is conserved, the ground state and the even excited state constitute a two-level system [18] that has been proposed as the basis for a new class of 
(a)

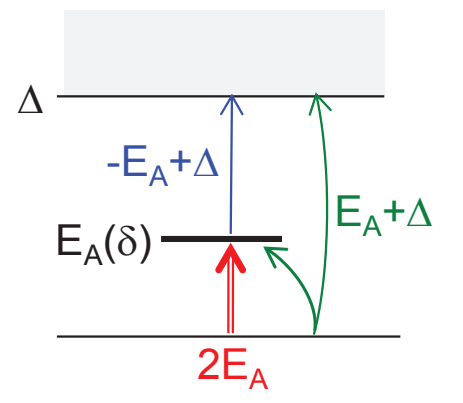

(b)

ground state

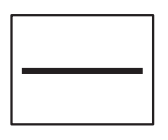

$-E_{A}(\delta)$

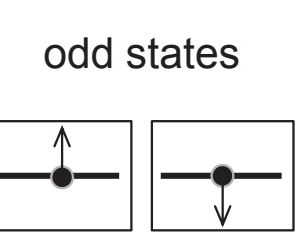

0 even

excited state

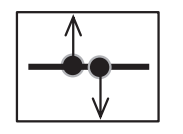

$\mathrm{E}_{\mathrm{A}}(\delta)$

Figure 1. (a) The single particle excitation spectrum for each channel of a short Josephson weak link consists of a doubly-degenerate Andreev level at energy $E_{A}(\delta)$, and a continuum of states at energies larger than the superconducting gap $\Delta$. Arrows indicate transitions that can be induced by microwave absorption. The four possible occupations of the Andreev level are shown in (b): they correspond to the ground state (Andreev level empty), the two odd states (a single quasiparticle in the Andreev level), and the excited pair state (doubly occupied Andreev level), with energies $-E_{A}$, 0 and $E_{A}$ respectively.

superconducting qubits [19, 20, 21, 22]. What is particularly interesting and novel is that in contrast with all other superconducting qubits based on Josephson junction circuits [23] an Andreev qubit is a microscopic two-level system akin to spin qubits in semiconducting quantum dots. Also, if one considers the odd states, despite the absence of actual barriers the system can be viewed as a superconducting "quantum dot" allowing manipulation of the spin degree of freedom of a single quasiparticle [24, 25, 26]. The coherence properties of Andreev doublets [20, 22] are still to be addressed experimentally. The relaxation time of the excited state and the dephasing time of quantum superpositions of the two states have to be measured, understood, and if possible, controlled.

Both relaxation and dephasing mechanisms contribute in principle to the linewidth of the Andreev transition. In order to achieve coherent manipulation of these Andreev states one would need much narrower lines than those observed in the aforementioned experiments, where they were typically larger than $500 \mathrm{MHz}$. This was most probably due to large superconducting phase fluctuations imposed by the dissipative measurement lines that were necessary to measure the current-voltage characteristics of the contacts, a key piece of information from which the $\left\{\tau_{i}\right\}$ are extracted. Here, to isolate efficiently the contact from external perturbations, we follow a strategy that has been implemented successfully for superconducting qubits [27, 28]. The idea is to include an atomic contact in a small superconducting loop to form an rf-SQUID inductively coupled to the electromagnetic field of a coplanar microwave resonator. The latter should act as a narrow-band filter to allow probing the Andreev transition at $2 E_{A}$ without excessive broadening. A similar setup was analyzed theoretically in [29], although here we have in addition avoided any galvanic connection of the SQUID loop with the rest of the circuit in order to minimize the probability of trapping out-of- 
equilibrium quasiparticles in the contact [17]. By varying the flux threading the SQUID loop the Andreev transition frequency can be brought into resonance with the resonator mode. This will result in hybridization of the Andreev levels and the cavity mode (see Figure 2). The goal of the experiment presented here is to perform spectroscopy of the Andreev levels of the contact as a first step towards coherent manipulation of the two-level system.
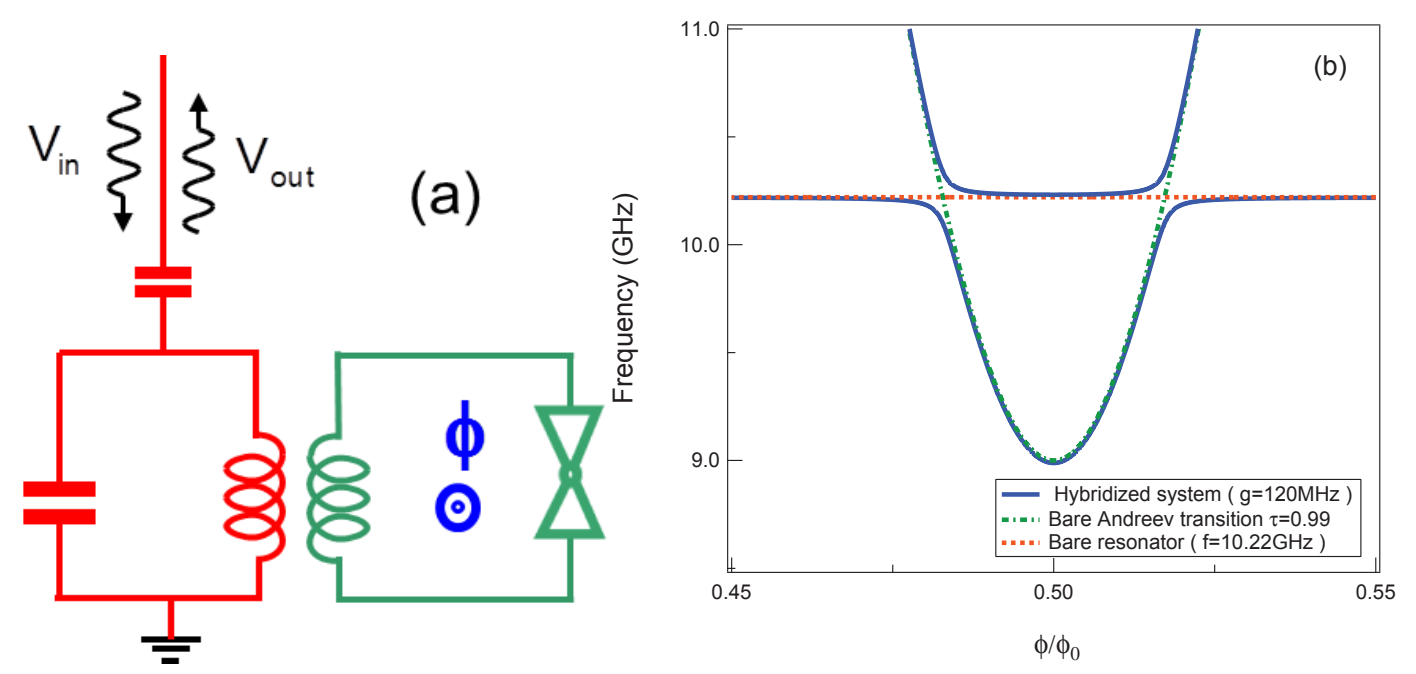

Figure 2. (a) An atomic rf-SQUID (in green) inductively coupled to a microwave resonator, represented here by a LC circuit (in red). The double triangle symbol represents the atomic contact. The spectrum of the combined system is probed through microwave reflectometry by weakly coupling the resonator to the external setup through a small capacitor. (b) example of the expected spectrum (blue full lines) as a function of the magnetic flux threading the SQUID loop. The resonance frequency of the bare resonator (red dashed line) is here $10.1 \mathrm{GHz}$; the Andreev frequency (green dashed line) corresponds to a channel with $\tau=0.991$; and the SQUID-resonator coupling energy is $h \times(120 \mathrm{MHz})$. The anti-crossing results from the hybridization of the two quantum systems.

\section{Experimental Methods}

\subsection{Sample fabrication}

The samples are fabricated on a flexible $500 \mu \mathrm{m}$-thick Kapton substrate $\left(\epsilon_{r} \simeq 3.2\right.$, $\tan \delta \sim 1 \times 10^{-4}$ at $\left.30 \mathrm{mK}\right), 50 \mathrm{~mm}$ in diameter. In a first step, a series of $\lambda / 4 \mathrm{Nb}$ resonators is fabricated. The substrate is then cut into $7 \mathrm{~mm} \times 16 \mathrm{~mm}$ chips which are individually processed afterward to fabricate the atomic SQUID.

2.1.1. The microwave resonator A $200 \mathrm{~nm}$ thick Nb layer sputtered over the whole substrate is patterned via optical lithography, and then structured using reactive ion etching into a series of quarter-wave $(\lambda / 4)$ resonators in a coplanar waveguide 
geometry (see Figure 3). The total length of the $36 \mu \mathrm{m}$ wide inner conductor is $5 \mathrm{~mm}$. The gap between the inner conductor and the ground plane is $18 \mu \mathrm{m}$. The impedance of the coplanar waveguide is $Z_{r} \sim 80 \Omega$. The resonator is coupled through an interdigitated capacitor $C \sim 5 \mathrm{fF}$ to a $50 \Omega$ line to measure its reflection coefficient $S_{11} \equiv 20 \log \left(V_{\text {out }} / V_{\text {in }}\right)$. For the $5 \mathrm{fF}$ coupling capacitor the external losses should dominate over the internal ones (arising essentially from dielectric losses in the kapton substrate) leading to a global quality factor on the order of 1000 .
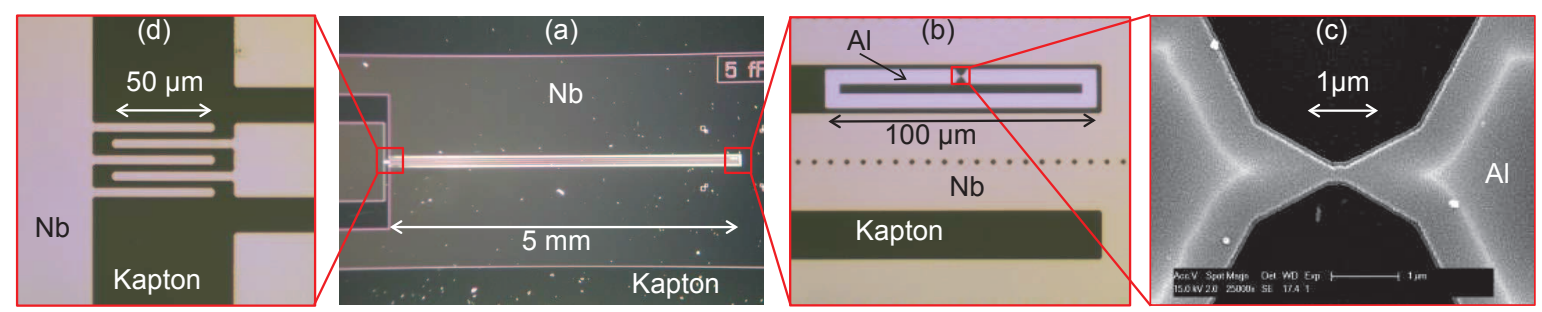

Figure 3. (a) Microphotograph of a coplanar quarter-wave $\mathrm{Nb}$ resonator. (b) Zoom on the shorted-end of the microwave $\mathrm{Nb}$ resonator (light gray). The $36 \mu \mathrm{m}-$ wide center line contains some small holes intended as pinning centers for eventual vortices trapped in the superconducting film. The aluminum loop (white), with a suspended microbridge in one arm, is placed within the $18 \mu \mathrm{m}$ gap (black). (c) Scanning electron microscope image of suspended microbridge with a $300 \mathrm{~nm}$-wide constriction. The bright $\mathrm{v}$-shaped ridges on both sides correspond to the border of the underlying polyimide layer which was etched to free the bridge over $\sim 2 \mu \mathrm{m}$. (d) interdigitated coupling capacitor of the resonator.

2.1.2. The atomic rf-SQUID. Using electron beam lithography we fabricate a $100 \mathrm{~nm}$ thick aluminum superconducting loop containing in one arm a micro-bridge, suspended over approximately $2 \mu \mathrm{m}$ by reactive ion etching of a sacrificial polyimide layer (Figure 3). The bridge has a $300 \mathrm{~nm}$ constriction at the center. The width and the inner dimensions of the loop are $5 \mu \mathrm{m}$ and $4 \mu \mathrm{m} \times 90 \mu \mathrm{m}$, respectively, which lead to a geometrical inductance of around $100 \mathrm{pH}$, much smaller than the Josephson inductance of a typical atomic contact (a few $\mathrm{nH}$ ). A magnetic flux $\phi$ through the loop is then used to impose a superconducting phase difference $\delta \cong 2 \pi \phi / \phi_{0}$ across the contact, where $\phi_{0}=h / 2 e$ is the flux quantum. This allows adjusting the phase-dependent energy of the Andreev levels.

\subsection{Setup}

Figure 4 shows the break-junction setup. The ensemble is attached to the mixing chamber of a dilution refrigerator. A precision screw (not shown), driven by a room temperature dc motor, controls the vertical displacement of a spindle. A copper slab attached to the spindle pushes the free end of the sample, which is firmly clamped on the opposite side against a microwave SMA launcher. The elongation of the upper face of the substrate as it bends leads eventually to the bridge rupturing. Afterward, the 
distance between the two resulting electrodes varies by a few tens of picometers for every micrometer of vertical displacement of the pusher. The temperature of the ensemble is below $100 \mathrm{mK}$, and the cryogenic vacuum ensures that there is no contamination of the freshly exposed electrodes. The electrodes are gently brought back together, reforming the bridge and creating an atomic-size contact. Contacts can be made repeatedly in order to vary the number of channels and/or the transmission probabilities. An important feature of the microfabricated break junctions [30] is that a given contact can be maintained for weeks with changes in transmission below one part in a thousand.

The sample holder is enclosed in a set of three cylindrical shields (Al, Cryoperm and $\mathrm{Cu}$, innermost to outermost) attached to the mixing chamber of a dilution refrigerator. All shields are capped at both ends. The inside diameter of the $\mathrm{Al}$ shield is $76 \mathrm{~mm}$. The intermediate Cryoperm shield diverts the ambient magnetic field to reduce flux fluctuations through the SQUID loop as well as to minimize the number of vortices trapped in the $\mathrm{Nb}$ superconducting film. The inner walls of the Al shield are covered with a $3 \mathrm{~mm}$ thick layer of epoxy loaded with bronze and carbon powder to damp cavity resonances and adsorb spurious infrared radiation [31, 32]. A small superconducting coil, placed $2 \mathrm{~mm}$ above the sample inside a copper shield, allows controlling the flux through the loop.

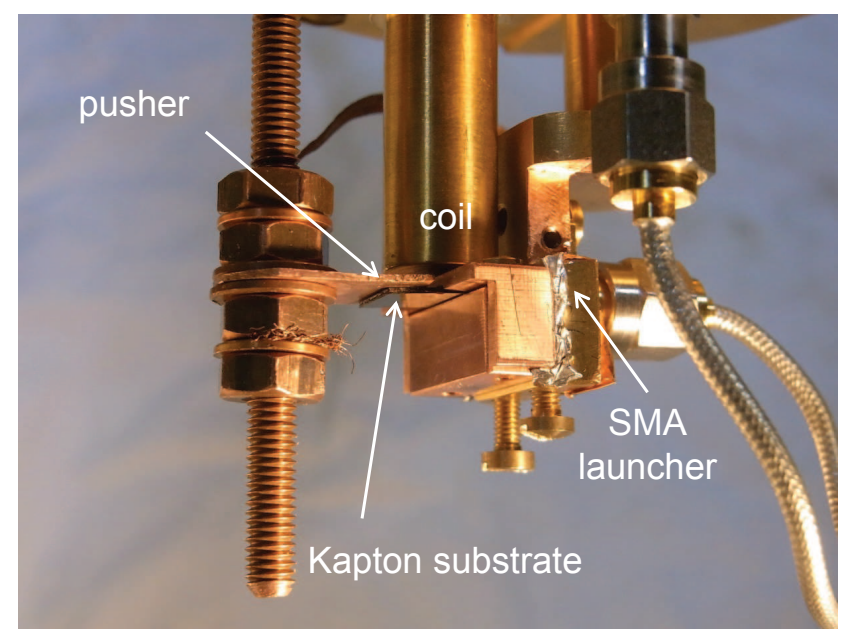

Figure 4. Break junction setup: a Kapton substrate is attached to SMA launcher (right side). The threaded spindle on the left side actuates the pusher vertically on the free end of the substrate with micrometer precision. The central cylindrical copper shield hosts a small superconducting coil that controls the flux through the SQUID loop. The whole system is enclosed in a set of three shields and anchored to the mixing chamber of a dilution refrigerator.

A single coaxial line enters this set of shields and connects to the SMA launcher. The overall microwave setup is sketched in Figure 5. There are two 8-12GHz circulators (Pamtech XTE0812KCSD) and one 0.1 - $18 \mathrm{GHz}$ directional-coupler (Clear Microwaves $\mathrm{C} 20218$ ) placed at the same temperature $(25 \mathrm{mK})$ as the sample but outside 
the shields. A first microwave tone, injected at one circulator, probes the response of the resonator at a frequency close to $\nu_{0}$. The reflected signal from the resonator goes through the two circulators into a cryogenic amplifier (0.5-11 GHz LNA \#265D from Caltech, gain $28 \mathrm{~dB}$ ) placed at $1 \mathrm{~K}$. To minimize losses in the return signal a superconducting coaxial cable (Coax-Co SC-086-50-NbTi-NbTi) is used between the second circulator and the cryogenic amplifier. The output line from the cryogenic amplifier to room temperature is a CuNi coax, with a silver cladded inner conductor (CoaX-Co SC-086/50$\mathrm{SCN}-\mathrm{CN})$. The two circulators prevent noise from the amplifier reaching the sample. A second tone at frequency $\nu_{1}$ can be injected through the directional-coupler $(-20 \mathrm{~dB}$ coupling) to drive the transition between the Andreev levels at the atomic contact. Each line has a series of attenuators placed at different stages of the refrigerator to prevent external noise from reaching the sample. The total attenuation of each of the two input lines (including losses in the cables) is $90 \mathrm{~dB}$.

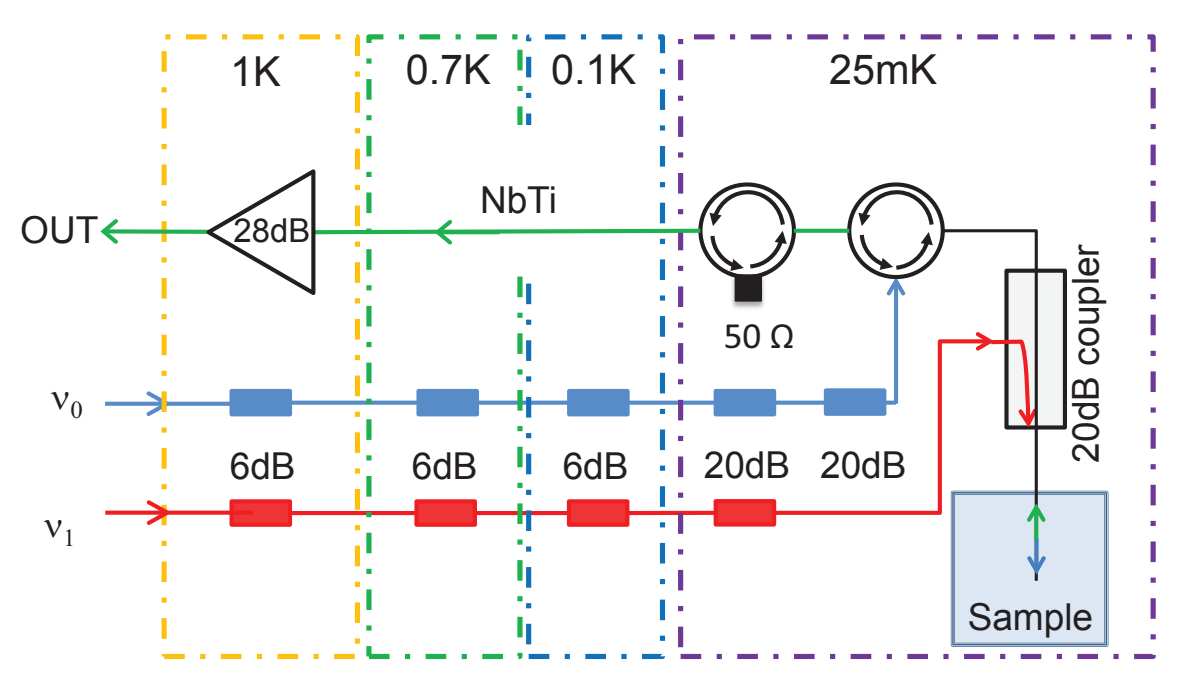

Figure 5. Microwave setup: A first tone of frequency $\nu_{0}$ is used to probe the resonator. The two circulators divert the reflected signal towards a $28 \mathrm{~dB}$ amplifier placed at $1 \mathrm{~K}$. A second tone of frequency $\nu_{1}$ is used to drive the Andreev transition of the atomic contact.

\section{Results}

\subsection{One-tone spectroscopy}

After an additional $78 \mathrm{~dB}$ room temperature amplification of the reflected signal, the reflection coefficient is directly measured using a vector network analyzer. As shown in Figure 6, three resonances appear in $S_{11}$ below $10 \mathrm{~K}$ in the range $8-10 \mathrm{GHz}$. Below the superconducting transition of aluminum, only the two lowest frequency lines (\#1 and \#2) depend on temperature and bending of the substrate, and are thus associated with on-chip modes. The coplanar mode resonance is the one at $\nu_{R} \sim 10.24 \mathrm{GHz}$, with a quality factor $Q \sim 300$. This is three times lower than expected. The measurements 
clearly indicate an undercoupled regime with only $40^{\circ}$ phase shift at resonance instead of the full $360^{\circ}$. We interpret this result as arising from the coupling of the coplanar resonator mode with a parasitic mode of the on-chip ground plane which is itself heavily damped by radiation to the enclosing dissipative cavity $\$$

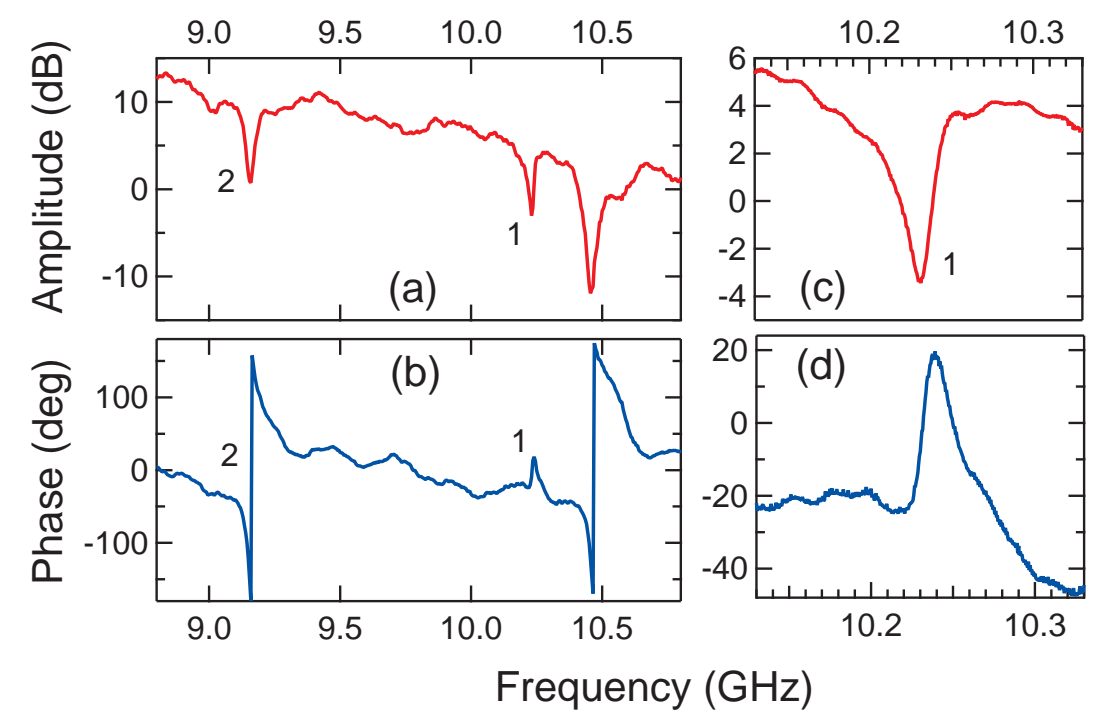

Figure 6. Amplitude (a) and phase (b) of reflection coefficient of resonator as a function of frequency, measured at $30 \mathrm{mK}$. The coplanar mode resonance is the one at $\nu_{R} \sim 10.24 \mathrm{GHz}$ (labeled $\# 1$ ). There are two parasitic resonances, at $9.16 \mathrm{GHz}$ and $10.45 \mathrm{GHz}$. Only resonances \#1 and \#2 change with temperature below $1 \mathrm{~K}$ and when bending the substrate and are thus associated to on-chip modes. (c) and (d): zooms around coplanar mode resonance.

Despite the low quality factor, it is still possible to probe the atomic SQUID. Figure $7 \mathrm{a}$ shows the evolution of the resonance frequency of the resonator as the substrate is slowly bent at low temperature. As the coplanar resonator elongates, its resonance frequency decreases with the pusher vertical position at a rate of approximately $50 \mathrm{kHz} / \mathrm{\mu m}$. The sharp frequency drop observed around a vertical deflection of $400 \mathrm{\mu m}$ signals the last stage of rupture of the break junction. The frequency shift is in agreement with the change in inductance expected when opening the SQUID loop. As the vertical displacement of the pusher is actually not measured in-situ, but

$\ddagger$ Because the sample must bent, the two outer electrodes of the coplanar resonator are actually grounded only at one end. As a result the ground plane behaves as an antenna. After carrying out the measurements we understood, through detailed electromagnetic simulations, that for the actual geometry of the resonator the quarter-wavelength mode had a similar resonance frequency than the antenna mode of the ground plane. The two modes hybridize and the resonator mode is also affected by radiation damping. Hybridization could be avoided by redesigning the resonator as a meander line (thus making the length of the ground plane electrode much smaller than the length of the resonator). 
deduced from the measured number of turns of the motor and the pitch of the screw, the backlash of the mechanical driving setup leads to a hysteresis of around $25 \mu \mathrm{m}$ between opening and closing directions. However, the position at which the abrupt frequency shift occurs is reproducible within a few microns for successive openings. In the region of this frequency drop the contact has atomic dimensions and its Josephson inductance becomes much larger than the geometrical inductance of the loop. In this limit, the resonator frequency evolves periodically with the magnetic flux threading the Al loop, as shown in Figure 7b. If the substrate is bent further, the loop opens completely and the flux modulation disappears.
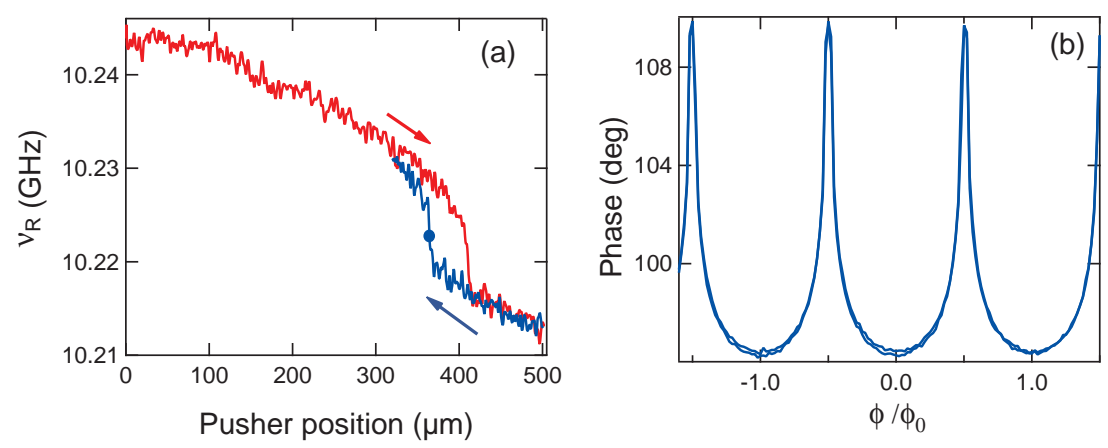

Figure 7. (a) Resonance frequency of resonator $\nu_{R}$ as a function of pusher position. Red (blue) curve correspond to the opening (closing) of the microbridge. (b) Phase of reflection signal as a function of the flux threading the SQUID loop (in reduced units) at the pusher position signaled by the blue dot in right panel. The modulation disappears when the microbridge is broken.

Figure 8 displays a spectrum of the reflection coefficient $S_{11}$ as a function of the probe frequency and the flux threading the loop. An anti-crossing between the resonator and an Andreev transition in the contact is clearly observed. As shown, the shape of the spectrum can be described, at least qualitatively, by considering a single channel of transmission $(\tau \sim 0.995)$ coupled $(g \sim 120 \mathrm{MHz})$ to the coplanar resonator harmonic oscillator [29].

\subsection{Two-tone spectroscopy}

The spectrum can be explored over a much wider frequency range by using a two-tone technique. In this case the resonator is constantly probed at a fixed frequency $\nu_{0}$ close to its resonance $\nu_{R}$, while sweeping the frequency $\nu_{1}$ of a second tone that is applied through the directional coupler (see Figure 5). The amplitude of this second tone is chopped at an audio frequency $\nu_{a}$. The reflected signal at $\nu_{0}$ is homodyne detected yielding the two quadratures I and $\mathrm{Q}$ which are then measured by lock-in amplifiers at $\nu_{a}$ (see Figure 9). 


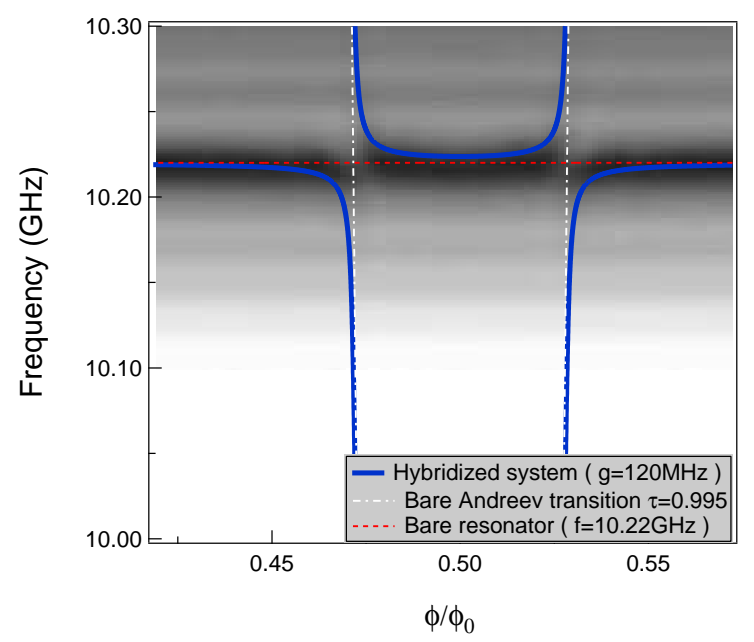

Figure 8. One-tone spectrum as a function of probe frequency (vertical axis) and phase across contact (horizontal). The gray level encodes the amplitude of the reflection coefficient. The dotted lines correspond to the bare frequencies for the resonator (red) and the Andreev transition of a single-channel of transmission $\tau \sim 0.995$ (white). The blue line is the prediction for the coupled system (coupling constant $g \sim 120 \mathrm{MHz}$ ).

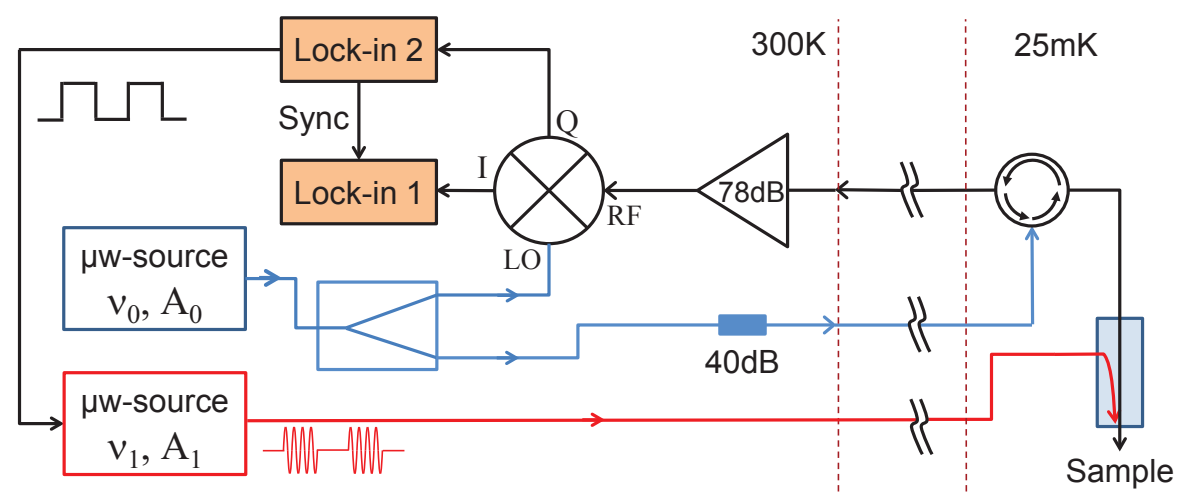

Figure 9. Room temperature setup for the two-tone spectroscopy: The pump signal at $\nu_{1}$ is chopped at audio frequency. The reflected signal at $\nu_{0}$ coming out of the cryostat is amplified and homodyne detected in a IQ mixer. The two phases are detected by the two lock-ins at the audio frequency at which the pump signal at $\nu_{1}$ is chopped.

The spectra of the reflected signal as a function of the flux through the loop (horizontal axis) and the frequency $\nu_{1}$ of the second tone (vertical axis) is shown in Figure 10 for two different contacts. Also shown in the figure are vertical cuts of each of the spectra at half flux quantum $(\delta=\pi)$, which are fitted using two lorentzian peaks, all with linewidths below $60 \mathrm{MHz}$. By fitting the spectra using the analytical expression for the Andreev transition frequency $2 E_{A} / h$, one can extract the gap $\Delta$ of the aluminum film and the transmission of the channels. Despite the apparently similar shapes of the multiple lines, the spectra do not correspond to contacts with several channels of slightly different transmissions, as shown by the continuous lines in 10(b). Instead, the 
appearance of several peaks is attributed to the high microwave power injected in order to acquire sufficient signal.
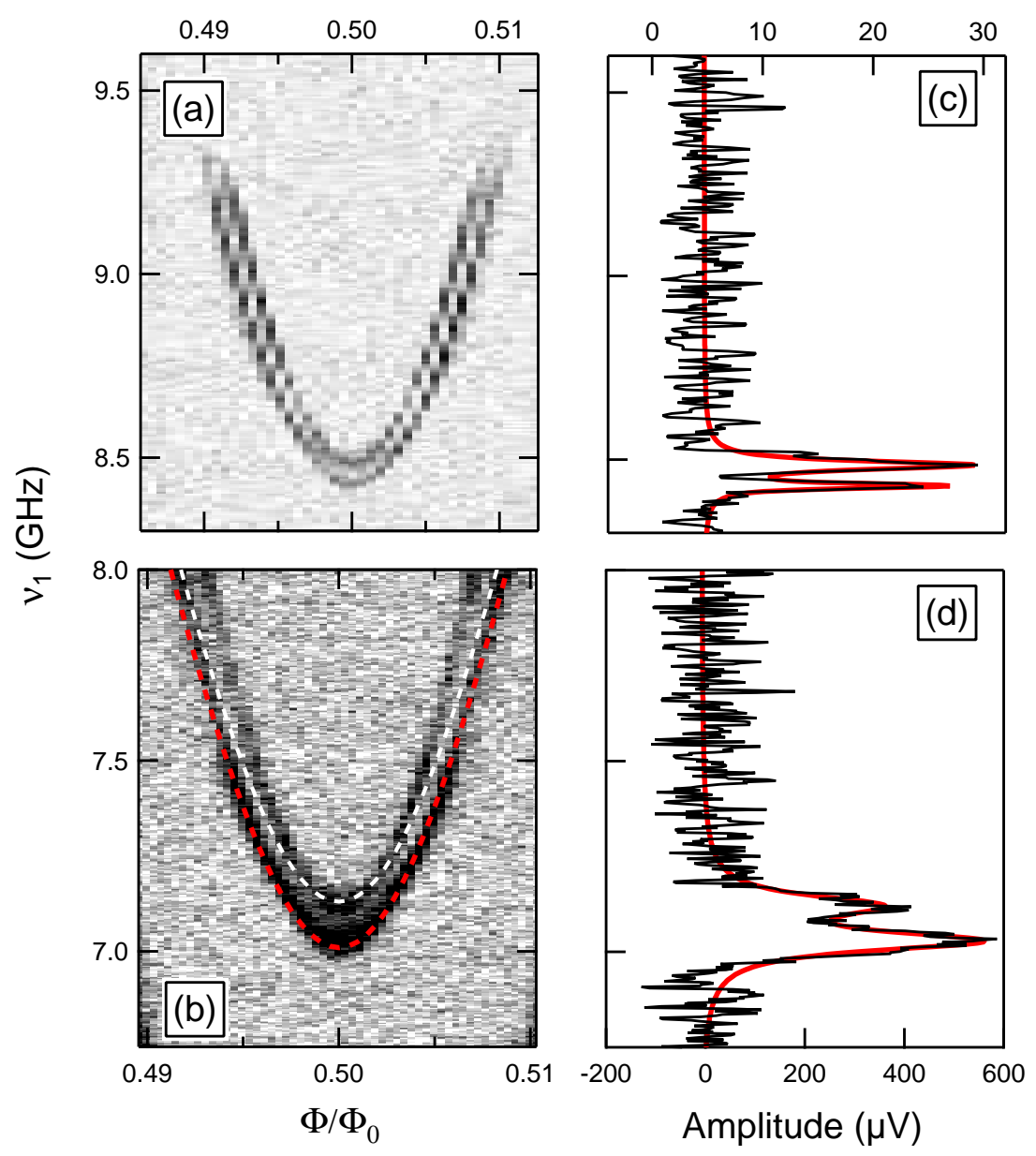

Figure 10. (a) and (b): Greyscale coded amplitude of the reflected signal at $\nu_{0}$, as a function of flux through the SQUID loop (horizontal) and frequency $\nu_{1}$ of second tone (vertical) for two different contacts. Fits like red dashed line in (b) using the analytical expression for the Andreev transition frequency $2 E_{A} / h$ determine the channel transmissions ( $\tau=0.9906$ in (a) and $\tau=0.9934$ in (b)). The white dashed line in (b) illustrates the fact that the additional features of the spectrum cannot be attributed to Andreev transitions of other channels with slightly different transmission coefficients. (c) Measured amplitude (black) of reflected signal at a flux $\phi=0.5 \phi_{0}$ for contact of panel (a). Red line: fit of measured data using two lorentzians of widths $17 \mathrm{MHz}$ and $28 \mathrm{MHz}$. (d) Measured amplitude (black) of reflected signal at a flux $\phi=0.5 \phi_{0}$ for contact of panel (b). Red line: fit of measured data using two lorentzians of widths $61 \mathrm{MHz}$ and $65 \mathrm{MHz}$. 


\section{Conclusions}

We have presented the first evidence for the efficient inductive coupling of a superconducting atomic contact to the electromagnetic field of a coplanar resonator. Using a two-tone setup, we have performed spectroscopy of the Andreev levels in the contact over several gigahertz. The observed linewidths are one order of magnitude smaller than in previous experiments and provide a lower bound of $10 \mathrm{~ns}$ for the coherence of the Andreev states. Although this is still too short for coherent manipulation of the Andreev doublet, we have identified a parasitic heavily damped resonance that loads the coplanar mode in the present design. A redesign of the resonator geometry to avoid this spurious resonance is expected to improve the lifetime by an order of magnitude. Pulsed pump and probe experiments should then allow performing Rabi oscillations of the state of the Andreev system.

\section{Acknowledgments}

We gratefully acknowledge technical support by P. Senat and P. F. Orfila, and the expert input and assistance of P. Bertet, D. Estève, P. Joyez and D. Vion. We have also greatly benefited from discussions with L. Bretheau, A. Levy Yeyati and J. M. Martinis. Financial support by ANR contracts DOCFLUC and MASH, C'Nano, the People Programme (Marie Curie Actions) of the European Union's Seventh Framework Programme (FP7/2007-2013) under REA grant agreement $n^{\circ}$ PIIF-GA-2011-298415, and ECOS-SUD (France-Argentina) $n^{\circ} \mathrm{A} 11 \mathrm{E} 04$ is acknowledged.

\section{References}

[1] N. Agraït, A. Levy Yeyati, and J. M. van Ruitenbeek, Physics Reports (2003), 81.

[2] E. Scheer, P. Joyez, D. Esteve, C. Urbina, and M. H. Devoret, Phys. Rev. Lett. 78, 3535 (1997).

[3] Yu. Nazarov and Ya. M. Blanter, "Quantum transport : introduction to nanoscience", Cambridge University Press (2009).

[4] R. Cron, M. F. Goffman, D. Esteve and C. Urbina, Phys. Rev. Lett. 86, 4104 (2001).

[5] R. Cron, E. Vecino, M.H. Devoret, D. Esteve, P. Joyez, A. Levy Yeyati, A. MartinRodero and C. Urbina, Proceedings of the XXXVIth Rencontres de Moriond « Electronic Correlations: From Meso- to Nano-physics », Les Arcs, (2001).

[6] L. Bretheau, Ç. Ö. Girit, L. Tosi, M. Goffman, P. Joyez, H. Pothier, D. Esteve, and C. Urbina, C. R. Physique 13, 89 (2012).

[7] B. D. Josephson, Phys. Lett. (1962), 251.

[8] A. A. Golubov, M. Yu. Kupriyanov, and E. Il'ichev Rev. Mod. Phys. 76, 411 (2004)

[9] A. Furusaki and M. Tsukada, Physica B: Condensed Matter (1990), 967-968. 
[10] C. W. J. Beenakker and H. van Houten, Phys. Rev. Lett. 66, (1991), 3056.

[11] L. Bretheau, Ç. Ö. Girit, H. Pothier, D. Esteve and C. Urbina, Nature 499, 312 (2013).

[12] L. Bretheau, PhD thesis (2013), available on-line at http://hal.archivesouvertes.fr/tel-00772851/

[13] L. Bretheau, Ç. Ö. Girit, C. Urbina, D. Esteve and H. Pothier, Phys. Rev. X 3, 041034 (2013).

[14] F. S. Bergeret, P. Virtanen, T. T. Heikkilä, and J. C. Cuevas, Phys. Rev. Lett. 105, 117001 (2010).

[15] F. S. Bergeret, P. Virtanen, A. Ozaeta, T. T. Heikkilä, and J. C. Cuevas, Phys. Rev. B 84, 054504 (2011).

[16] F. Kos, S. E. Nigg, and L. I. Glazman, Phys. Rev. B 87, 174521 (2013).

[17] M. Zgirski, L. Bretheau, Q. Le Masne, H. Pothier, D. Esteve, and C. Urbina, Phys. Rev. Lett. 106, 257003 (2011).

[18] D. A. Ivanov and M. V. Feigel'man, Phys. Rev. B 59, 8444 (1999).

[19] J. Lantz, V. Shumeiko, Bratus, E. and Wendin, G., Physica C: Superconductivity (2002), 315 .

[20] M. A. Desposito and A. Levy Yeyati, Phys. Rev. B 64, 140511 (2001).

[21] A. Zazunov, V. S. Shumeiko, E. N. Bratus', J. Lantz, and G. Wendin, Phys. Rev. Lett. 90, 087003 (2003).

[22] A. Zazunov, V. S. Shumeiko, G. Wendin, and E. N. Bratus, Phys. Rev. B 71, 214505 (2005).

[23] G. Wendin and V. S. Shumeiko, Low Temp. Phys. 33, 724 (2007).

[24] N. M. Chtchelkatchev and Yu.V. Nazarov, Phys. Rev. Lett. 90, 226806 (2003).

[25] J. Michelsen, V. S. Shumeiko, and G. Wendin, Phys. Rev. B 77, 184506 (2008).

[26] C. Padurariu and Yu. V. Nazarov, EPL 100, 57006 (2012).

[27] A. Blais, Ren-Shou Huang, A. Wallraff, S. M. Girvin, and R. J. Schoelkopf, Phys. Rev. A 69, 062320 (2004).

[28] A. Blais, J. Gambetta,1A. Wallraff, D. I. Schuster, S. M. Girvin, M. H. Devoret, and R. J. Schoelkopf, Phys. Rev. A 75, 032329 (2007).

[29] G. Romero, I. Lizuain, V. S. Shumeiko, E. Solano, and F. S. Bergeret, Phys. Rev. B 85, 180506 (2012).

[30] J.M. Van Ruitenbeek, A. Alvarez, I. Pineyro, C. Grahmann, P. Joyez, M. Devoret, D. Esteve and C. Urbina, Rev. Sci. Instrum. 67, 108 (1996).

[31] R. Barends, J. Wenner, M. Lenander, Y. Chen, R. C. Bialczak, J. Kelly, E. Lucero, P. O'Malley, M. Mariantoni, D. Sank, H. Wang, T. C. White, Y. Yin, J. Zhao, A. N. Cleland, J. M. Martinis, and J. J. A. Baselmans, Appl. Phys. Lett. 99, 113507 (2011). 
[32] A. D. Córcoles, J. M. Chow, J. M. Gambetta, C. Rigetti, J. R. Rozen, G. A. Keefe, M. B. Rothwell, M. B. Ketchen and M. Steffen, Appl. Phys. Lett. 99, 181906 (2011). 